\title{
Uncoupling protein 2 regulates myocardial apoptosis via the diabetogenic action of streptozotocin
}

\author{
Xiu-Zhen Li ${ }^{1}$, Ruo-Yun Tan', Xiang Lu ${ }^{1 *}$ \\ ${ }^{1}$ The First Clinical College, NJMU, Nanjing, China; \\ ${ }^{2}$ The Second Clinical College, NJMU, Nanjing, China. \\ Email: luxiang66@hotmail.com, lixiuzhen1985@163.com
}

Received 10 January 2010; revised 26 April 2010; accepted 24 May 2011.

\begin{abstract}
Objective: Determine the role of uncoupling protein 2 (UCP2) in the myocardial apoptosis of diabetic mellitus ( DM ) . Methods: DM animal models were induced by streptozotocinon (STZ) on UCP2 knock-out mice (UCP2KO) and wild-type mice (WT), which were reared for 7 and 28 days after successful modeling, respectively. The expressions of relative protein for myocardial apoptosis, pro-caspase-9, were investigated using western blot. However, the terminal deoxynucleotidyltransferase-mediated dUTP-biotin nick end labeling (TUNEL) was used to explain apoptosis at the DNA level. Results: Image analysis showed that the expression of pro-caspase-9 protein levels increased slightly in UCP-/- + DM-7-day group comparing with DM-7-day group $(P>0.05)$. The expression of pro-caspase-9 protein levels increased significantly $(P$ $<0.05$ ) in UCP-/- + DM-28-day group comparing with DM-28-day group. TUNEL analysis indicated that UCP2 reduced the number of apoptotic myocytes in the DM-28-day group by $70 \%$ in comparison to DM-7-day group by $30 \%(P<0.05)$. Conclusion UCP2 may be one of the most important factors that contribute to the myocardial apoptosis of DM.
\end{abstract}

Keywords: Uncoupling Protein 2; Diabetes; Myocardium; Apoptosis

\section{INTRODUCTION}

An inner mitochondrial protein known as uncoupling protein 2 (UCP2) is a member of the uncoupling protein family and belongs to the inner mitochondrial membrane anion-carrier superfamily. UCP2 can be detected in the heart [1], brain, lung, spleen, kidney, liver, and adipose tissue. $\mathrm{UCP} 2$ is a regulator of reactive oxygen species during electron transport in the mitochondrial inner membrane, plays an active role in the prevention of atherosclerosis [2], is one of the etiologies of type 2 diabetes [3], participates in inflammation [4], and regulates cell apoptosis [5] and aging [6]. The purpose of this study was to investigate the relationship between UCP2 and the myocardial apoptosis of diabetic mellitus, demonstrate the important role of UCP2 in diabetic cardiomyopathy (DC), and provide additional theoretical foundation for DC therapy.

\section{MATERIALS AND METHODS}

\subsection{Animals}

Male, 8- to 12-week old WT and UCP2KO littermate mice were used in this study. UCP2KO and WT mice were generated on a C57B/6 mixed background. The WT mice were purchased from the Shanghai Silaike Laboratory Animal Company. UCP2KO mice were previously generated by homologous recombination in embryonic stem cells on a C57BL/6 mixed background in the Laboratory Animal Center of Nanjing University, wherein their genomic loci were analyzed using PCR, as previously described [7]. The WT and UCP2KO mice were raised in a colony at the Nanjing Medical University, wherein all of the animal protocols were approved by the Animal Care Committee, and the animals were handled according to the guidelines of the Chinese Council of Animal Care.

\subsection{Chemicals and Reagents}

STZ (N-(methylnitrosocarbamoyl)- $\alpha$-D-glucosamine), anti-caspase9 antibody, and a TUNEL Apoptosis Assay Kit was obtained from Bioworld.

\subsection{STZ-Induced Diabetes}

Each mouse received a single daily injection of STZ (60 $\mathrm{mg} / \mathrm{kg}$ ) for three consecutive days. Animals in the negative control group (NC) and blank control group (BC) were identically handled, but were injected with only vehicle (citrate buffer). All STZ-Injected animals were assessed for the development of diabetes as described previously [8] and sacrificed on days 7 or 28 , and their tissues were harvested for further analyses. 


\subsection{Blood Glucose Concentration Measurements}

Plasma glucagon concentrations were assayed using a glucometer (Johnson \& Johnson) according to the manufacturer's instructions.

\subsection{Western Blotting}

Heart tissues were immediately homogenized in Lysis Buffer at $4^{\circ} \mathrm{C}$ as described previously [9]. Insoluble material was removed by centrifugation for $30 \mathrm{~min}$ at $14,000 \mathrm{rpm}$ at $4^{\circ} \mathrm{C}$. The protein concentration of apoptosis protein was measured using a bicinchoninic acid (BCA) kit and standardized for $60 \mu \mathrm{g} / \mathrm{ml}$. The total protein was separated by $12 \%$ separating gel with a $3.9 \%$ stacking gel. Next, the gel was electro transferred onto nitrocellulose membranes (Trans-Blot, Bio-Rad) and blocked with 5\% skim milk for $2 \mathrm{~h}$. The membrane was incubated in primary antibody (anti-pro-caspase-9, 1:600) and diluted in $0.1 \%$ Tween Tris-buffered saline (TBST) overnight at $4^{\circ} \mathrm{C}$. Subsequently, the membrane was incubated in secondary antibody (anti-rabbit IgG HRP conjugate, 1:800) and diluted in $0.1 \%$ TBST for $1 \mathrm{~h}$ at room temperature. Specific signals were detected using enhanced chemiluminescent (ECL) plus reagent (Bio$\mathrm{Rad})$. The protein loading was $30 \mu \mathrm{l}$ and was normalized using a housekeeping gene antibody (mouse anti $\beta$-actin at 1:10000 and anti-mouse IgG HRP at 1:5000).

\subsection{Apoptosis and Immunohistochemistry}

Small $(<1.0 \mathrm{~cm})$ pieces of heart remnants were fixed overnight at $4{ }^{\circ} \mathrm{C}$ in a solution of $4 \%$ paraformaldehyde in phosphate-buffered saline. The tissue was dehydrated, paraffin-embedded, sliced (4- $\mu \mathrm{m}$ thickness), dewaxed, and hydrated. Apoptosis was assessed via the TUNEL method using a ApopTag fluorescein in situ detection kit (Bioworld). Labeling indices were determined without previous knowledge of the genotype of the mice by counting the number of TUNEL-positive cells per 40 myocytes in each field. There were at least five fields for each slice and six slices for each animal.

\section{STATISTICAL ANALYSIS}

The data are expressed as mean \pm S.E.M, where applicable. The significance of the data was evaluated using univariate analysis via the Tukey-Kramer test or Dunn's multiple comparisons post test, where applicable. Differences were considered as being statistically significant at $P<0.05$.

\section{RESULTS}

\subsection{The Effect of UCP2 on the Development of Hyperglycemia in STZ-Treated Mice}

WT and UCP2KO were compared to evaluate how the absence of UCP2 affects the development of hyperglycemia. Blood glucose was measured at the time of successful modeling, in order to monitor the development of the STZ-induced hyperglycemia. Both control (normal saline only) groups exhibited consistent blood glucose homeostasis over the 7-day and 28-day study periods and did not show any significant difference or change in blood glucose concentration in comparison to their starting values. After successful modeling, the blood glucose levels in both the WT and UCP2KO mice were not significantly different; however, after day 28 , STZtreated UCP2KO mice demonstrated less hyperglycemia development in comparison to WT mice (Figure 1).

\subsection{The Effect of UCP2 on Myocardial Apoptosis after STZ Treatment}

Myocardial apoptosis was measured via Western blot analysis of apoptotic protein and TUNEL staining on paraffin sections.

The expression of pro-caspase-9 was observed to increase in the UCP-/- + DM-7-day group in comparison to the DM-7-day group, although this increase was not statistically significant $(P>0.05)$, whereas the expression of pro-caspase- 9 was observed to increase in the UCP2-/- + DM-28-day group in comparison to the DM-28-day group, wherein these results were statistically significant $(P<0.05$, Figure 2$)$. The number of TdT-labeled myocytes decreased 2.0-fold, from $71.00 \pm$ $0.087 \%$ in the UCP2KO + DM-28-day group to $30.10 \pm$ $0.079 \%$ in the DM-28-day group $(P<0.05)$, whereas decreased 1.2-fold, from $12.98 \pm 0.050 \%$ in the $\mathrm{UCP} 2 \mathrm{KO}+\mathrm{DM}-7$-day group to $9.84 \pm 0.079 \%$ in the DM-7-day group $(P>0.05)$ (Figure 3$)$.

\section{DISCUSSION}

DM is a major risk factor for the development of several cardiovascular complications, which in turn have be-

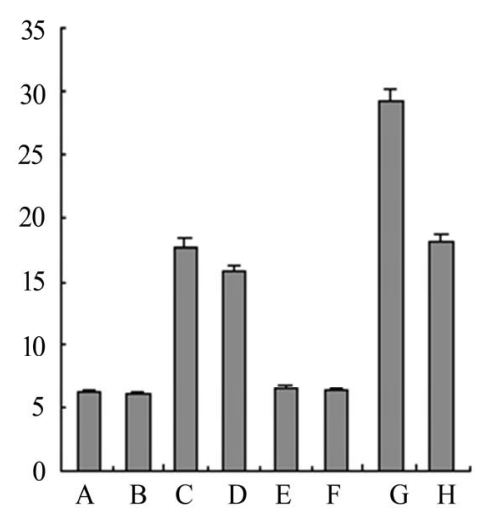

Figure 1. Blood glucose levels in the different groups, for A, B, C, D, E, F, $\mathrm{G}$. 


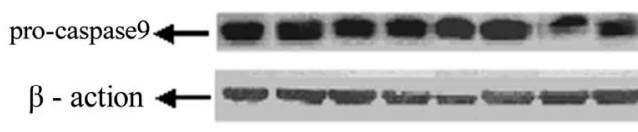

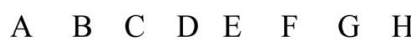

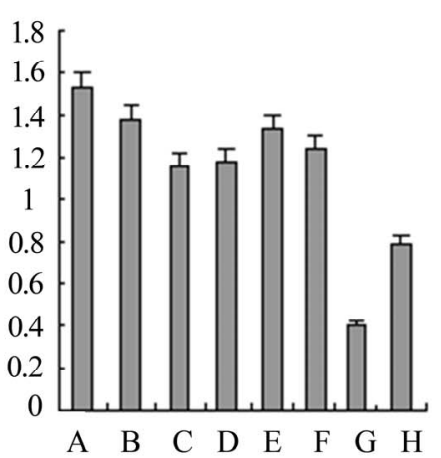

Figure 2. The expression of pro-caspase9 at different time (7-day, 28-day) in different groups, for A, B, C, D, E, F, G.

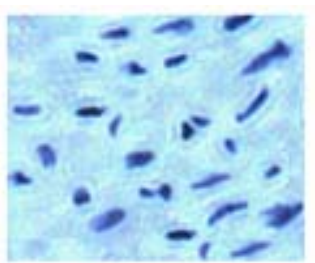

(a)

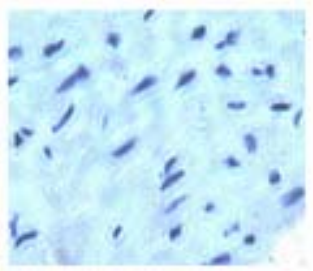

(e)

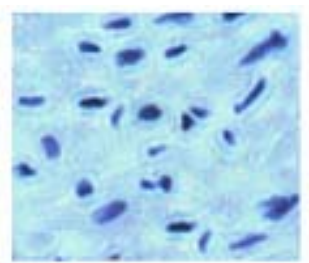

(b)

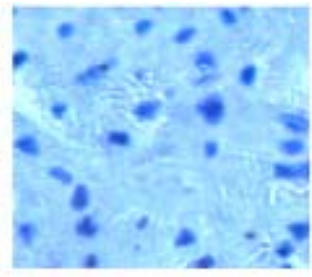

(f)



(c)

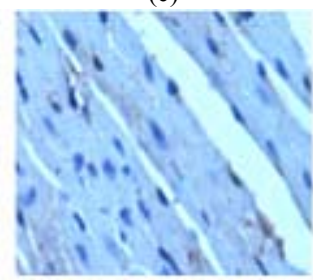

(g)

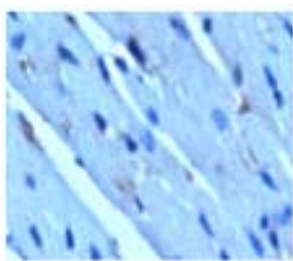

(d)

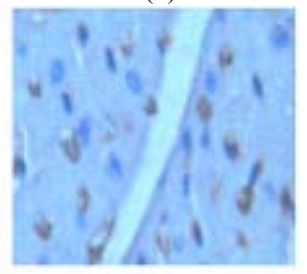

(h)

Figure 3. The result of TUNEL $(10 \times 40)$, respectively, A: NC7-day; B: DM7-day; C: U-/-7-day; D: UCP2-/- + DM7-day E:NC28-day; F: DM28-day; G: U-/-28-day; H: UCP2-/- + DM28-day; *P $<0.05$ versus wild-type.

come the primary causes of death in the diabetic population [10] Hyperglycemia is considered to be the primary cause of most chronic diabetic complications, such as cardiovascular pathological changes, diabetic retinopathy, diabetic nephropathy, diabetic foot, etc., which occurs via the deregulation of $\beta$-cell insulin secretion and the development of peripheral tissue insulin resistance.

DC was first reported in 1972 by Rubler et al. [11], who reported the autopsy data obtained from four patients with diabetic renal microangiopathy and dilated left ventricles in the absence of other common causes. Therein, they observed that there was a close relationship between myocardial apoptosis and the occurrence or development of diabetic cardiomyopathy. In the mitochondrial-initiated pathway, mitochondrial cytochrome c release and the activation of caspase play important roles in diabetes-induced cardiac cell death. Caspase activation is triggered by the formation of a multimeric Apaf-1/cytochrome c complex that is fully functional in the recruitment and activation of pro-caspase-9, resulting in further apoptosis.

In order to further investigate the role of UCP2 in the development of diabetes, we treated both WT and UCP2KO mice with STZ injections as to experimentally induce diabetes. We observed that the UCP2KO mice developed hyperglycemia, although significantly less severe in comparison to the WT group.

After four weeks, the myocardial mitochondria of DM mouse began to swell and denature [12]; On days 7, 14, and 21 after a single dose of STZ $(150 \mathrm{mg} / \mathrm{kg})$ treatment, the number of TUNEL-positive cells and the amount of caspase-3 activation increased [13]; Twenty-eight days after successful modeling (STZ treatment, $60 \mathrm{mg} / \mathrm{kg}$ ), cardiac apoptosis in the Sprague Dawley (SD) rats were observed to have increased $(P<0.01)[14]$; The number of cardiac myocytes, which cultured by different blood glucose level, associated with blood glucose concentration; and Hyperglycemia through mitochondrial pathway resulted in myocardial apoptosis [15]. Our previous work has indicated that, even though the expression of 
cleaved-caspase-3 increased, in addition to cell shrinkage and nuclear condensation in the DM-7-day group in comparison to the WT group, this increase was not statistically significant $(P>0.05)$, which is consistent with our reported experimental data.

UCP2 plays a significant role in the onset of diabetes, specifically via the regulation of cellular ATP production [16], islet beta-cell apoptosis [17] and reduction ROS production [18]. UCP2 also affects the occurrence or development of DM and participates in the myocardial apoptosis of DC. UCP2KO developed lesser hyperglycemia than their WT counterparts after multiple low-dose STZ injections in vivo [19]. TUNEL analysis indicated that the number of apoptotic myocytes in the $\mathrm{UCP} 2 \mathrm{KO}+\mathrm{DM} 28$-day group decreased by $30 \%$ in comparison to in the DM-28-day $(P<0.05)$, while the number of apoptotic myocytes in the UCP2KO + DM7-day group decreased by $0.2 \%$ in comparison to the DM-7-day $(P>0.05)$. Our previous work has indicated that cleaved-caspase- 3 expression markedly decreases in UCP2KO + DM-28-day mice, wherein chromatin was condensed with high intensity fluorescence in comparison to DM-28-day mice, with a statistical significance ( $P$ $<0.05$ ); however, the expression of the cleaved-caspase-3 slightly decreased in the UCP2-/- + DM-7-day group, wherein chromatin was condensed with low intensity fluorescence, in comparison to the DM-7-day group, although the results were not statistically significant $(P>0.05)$. Therefore, it can be concluded that UCP2 reduces the number of apoptotic myocytes and can provide time-dependent anti-apoptosis activity via variable caspase- 3 or caspase- 9 expression.

In this article, myocardial apoptosis slightly increased in the negative control group in comparison to the blank group, although this increase was not statistically significant $(P>0.05)$. Although cardiac mitochondrial UCP2-mediated uncoupling respiration was impaired, short-term mitochondrial efficacy was increased, which (7-day or 28-day) improved the function of the myocardium.

Diabetic cardiomyopathy results in myocardial dysfunction. Meanwhile, it has been reported that the loss of myocardial cell quality and quantity could act as an important factor in heart dysfunction because they are non-renewable. UCP2 plays a crucial role in mitochondrial energy metabolism and in the occurrence or development of DC. Furthermore, it is reported that UCP2 is involved in determining lifespan [20]. Further research into UCP2 should investigate the relationship between UCP2 and myocardial apoptosis, and therein, attempt to identify the causes so as to improve treatment protocols and provide a more developed basis for the diagnosis and treatment of DC.

\section{REFERENCES}

[1] Ricquier, D. and Bouillaud, F. (2000) The uncoupling protein homologues: UCP1, UCP2, UCP3, StUCP and AtUCP. Biochemical Journal, 345, 161-179. doi:10.1042/0264-6021:3450161

[2] Blanc, J., Alves-Guerra, M.C., Esposito, B., et al. (2003) Protective role of uncoupling protein 2 in atherosclerosis. Circulation, 107, 388-390. doi:10.1161/01.CIR.0000051722.66074.60

[3] Chan, C.B., Saleh, M.C., Koshkin, V., et al. (2004) Uncoupling protein 2 and islet function. Diabetes, 53, 136-142. doi:10.2337/diabetes.53.2007.S136

[4] Arsenijevic, D., Onuma, H., Pecqueur, C., et al. (2000) Disruption of the uncoupling protein-2 gene in mice reveals a role in immunity and reactive oxygen species production. Nature Genetics, 26, 435-439. doi:10.1038/82565

[5] Teshima, Y., Akao, M., Jones, S.P., et al. (2003) Uncoupling protein-2 overexpression inhibits mitochondrial death pathway in cardiomyocytes circulation research. Circulation Research, 93, 192-200。 doi:10.1161/01.RES.0000085581.60197.4D

[6] Brand, M.D., Buckingham, J.A., Esteves, T.C., et al. (2004) Mitochondrial superoxide and aging: Uncoupling-protein activity and superoxide production. Biochemical Society Symposia, 71, 203-213.

[7] Zhang, C., Baffy, G., Perret, P., et al. (2001) Uncoupling protein-2 negatively regulates insulin secretion and is a major link between obesity, $\beta$-cell dysfunction, and type 2 diabetes. Cell, 105, 745-755.

doi:10.1016/S0092-8674(01)00378-6

[8] King, L.M., Sidell, R.J., Wilding, J.R., Radda, G.K. and Clarke, K. (2001) Free fatty acids, but not ketone bodies, protect diabetic rat hearts during low-flow ischemia. American Journal of Physiology-Heart and Circulatory Physiology, 280, H1173-H1181.

[9] Li, Z.H., Zhang, T., Dai, H.Y., et al. (2007) Involvement of endoplasmic reticulum stress in myocardial apoptosis of streptozocin-induced diabetic rats. Journal of Clinical Biochemistry and Nutrition, 41, 58-67. doi:10.3164/jcbn.2007008

[10] Devereux, R.B., Roman, M.J., Paranicas, M., et al. (2000) Impact of diabetes on cardiac structure and function: The strong heart study. Circulation, 101, 2271-2276.

[11] Rubler, S., Dlugash, J., Yuceoglu, Y.Z., et al. (1997) New type of cardiomyopathy associated with diabetic Glomerulosclerosis. American Journal of Cardiology, 30, 595-602. doi:10.1016/0002-9149(72)90595-4

[12] Wei, X.X., Zhou, X.P., He, D., et al. (1992) Cardiac disease in streptozocin-induced diabetes. Chinese Journal of Endocrinology and Metabolism, 4, 222-225.

[13] Cai, L., Wang, Y., Zhou, G., et al. (2006) Attenuation by metallothionein of early cardiac cell death via suppression of mitochondrial oxidative stress results in a prevention of diabetic cardiomyopathy. Journal of the American College of Cardiology, 48, 1688-1697. doi:10.1016/j.jacc.2006.07.022

[14] Sung, P.H., Sun, C.K., Ko, S.F., et al. (2009) Impact of hyperglycemic control on left ventricular myocardium a 
molecular and cellular basic study in a diabetic rat mode. International Heart Journal, 50, 191-206. doi:10.1536/ihj.50.191

[15] Li, C.Y., Cao, L.S., Zeng, Q.T., et al. (2005) Taurine may prevent diabetic rats from developing cardiomyopathy also by down regulating angiotensin II type-2 receptor expression. Cardiovascular Drugs and Therapy, 19, 105-112. doi:10.1007/s10557-005-0443-X

[16] Joseph, J.W., Koshkin, V., Zhang, C.Y., et al. (2002) Uncoupling protein 2 knockout mice have enhanced insulin secretary capacity after a high-fat diet. Diabetes, $\mathbf{5 1}$, 3211-3219. doi:10.2337/diabetes.51.11.3211

[17] Joseph, J.W., Koshkin, V., Saleh, M.C., et al. (2004) Free fatty acid-induced beta-cell defects are dependent on uncoupling protein 2 expressions. The Journal of Biological Chemistry, 279, 51049-51056.
doi:10.1074/jbc.M409189200

[18] Arsenijevic, D., Onuma, H., Pecqueur, C., et al. (2000) Disruption of the uncoupling protein gene in mice reveals a role in immunity and reactive oxygen species production. Nature Genetics, 26, 435-439. doi:10.1038/82565

[19] Lee, S.C., Robson-Doucette, C.A. and Wheeler, M.B. (2009) Uncoupling protein 2 regulates reactive oxygen species formation in islets and influences susceptibility to diabetogenic action of streptozotocin. Journal of Endocrinology, 203, 33-43. doi:10.1677/JOE-09-0117.

[20] Andrews, Z.B. and Horvath, T. (2009) Uncoupling protein-2 regulates lifespan in mice. American Journal of Physiology-Endocrinology and Metabolism, 296, E621-E627. doi:10.1152/ajpendo.90903.2008 